Marsha M. Cohen MD FRCPC

Peter G. Duncan MD FRCPC

William D.B. Pope MD FRCPC

Christopher Wolkenstein MBBS FRCPC

Departments of Anaesthesia and Social and Preventive

Medicine, Faculty of Medicine

University of Manitoba

750 Bannatyne Avenue

Winnipeg, Manitoba

R3E 0W3

REFERENCE

1 Beard $K$, Jick $H$, Walker AM. Adverse respiratory events occurring in the recovery room after general anesthesia. Anesthesiology 1986; 64: 269-72.

\section{Consent for epidural anaesthesia}

To the Editor:

The letter by Slusarenko and Noble regarding information given to patients to enable them to give informed consent for epidural anaesthesia is timely. Many departments of anaesthesia are wrestling with the same problem. Following this letter is the text of an information sheet about epidural analgesia that is given to patients having deliveries in the Dr. Everett Chalmers Hospital, a tertiary obstetrical unit.

The information sheet is given to the patient by the obstetrician toward the end of the pregnancy, along with other information regarding her anticipated delivery. The sheet is also available in the obstetrical unit for patients who did not receive it prior to hospital admission. This approach is not the final answer to informing our patients but we feel that it is a step in the right direction.

The initial experience (since January 1986) with the information sheet has been very positive. Most patients seem to appreciate the specific information plus the opportunity to ask questions. To our knowledge, only one patient has refused an epidural anaesthetic because of the infomation sheet. We plan to conduct a prospective examination of the value of the information sheet.

John Price MB FRCPC

Department of Anaesthesia

The Dr. Everett Chalmers Hospital

Fredericton, New Brunswick

E3B 5N5
Department of Anaesthesia

Information about epidural analgesia

All women experience a certain amount of discomfort during labour and delivery. Some women experience extremely severe pain and may require large doses of pain relieving drugs.

If it is indicated, we may be able to relieve your pain by "freezing" the nerves that carry the pain sensation from your womb. This is done by placing the freezing drugs around the nerves in the spinal canal, using an epidural catheter. This is a small tube placed in your back, outside the spinal cord. This procedure is performed by an anaesthetist who is requested by your obstetrician to do so.

The procedure is normally a perfectly safe way of providing pain relief. However, like all medical procedures, it carries with it the risk of certain complications, which usually cannot be anticipated.

These complications are not usually life-threatening, provided they are recognized and treated immediately. Your anaesthetist, who is a specialist physician, is trained to manage all of the complications associated with this procedure, just as your obstetrician will recognize and treat complications that may arise during your pregnancy and labour.

Certain wornen may not be suitable candidates for epidural analgesia. Occasionally (one per cent) the epidural catheter enters the space around the spinal cord. If this is not recognized, anaesthesia to a higher level in the body than is needed can occur. This is not a serious complication provided it is treated appropriately.

If the needle that is used to place the catheter or the catheter itself enters the space around the spinal cord, the patient may experience a headache in the days following delivery. Certain things can be done to reduce the likelihood of a severe headache if the situation arises.

Some women experience backache after having an epidural. This is the result of bruising of the tissues during the placement of the catheter in the epidural space. It may last for up to 14 days but is not disabling.

Very occasionally, the freezing may enter directly into a vein. If this occurs, you will notice a peculiar sensation in your head. Your ears will ring and you will have a "tingling" feeling in your face- You may also have a metallic taste in your mouth and throat. If this occurs, inform your nurse immediately.

If you are having an epidural for labour and delivery, you may have a continuous infusion of the freezing drug into the epidural catheter. You will experience relief of your pain but you will still be able to move. In fact, you will be required to move yourself from side to side every so often. If you cannot do this, your nurse should be notified immediately.

Other complications may arise which are more severe, but which occur very rarely. The most 
serious of these, as with any medical procedure, is of course, death. This is an extremely rare complication and occurs in less than 1 in 100,000 cases. Similarly, and only slightly more frequently, permanent nerve damage can occur. We are prepared to discuss all these complications with you if you wish. You must understand that all precautions are taken to avoid complications and to ensure that you and your baby are safe during your labour and delivery.

After you have read this pamphlet, if you wish to discuss any points, please indicate to your obstetrician and he will make arrangements for you to meet with an anaesthetist. You will be required to sign a form indicating that you consent to having the procedure performed and that you have been informed of the risks of the procedure, and have had an opportunity to ask questions of an anaesthetist.

Please understand that the final decision as to whether or not you have an epidural anaesthetic rests with you and your anaesthetist who is performing the procedure. He will base his decision on what he feels is best for you and your baby after consultation with your obstetrician.

Department of Anaesthesia

Dr. Everett Chalmers Hospital

Hôpital Dr. Everett Chalmers

\section{Obstruction of fresh gas flow in an Ayre's T-piece}

To the Editor:

We would like to report an incident of fresh gas flow failure in an Ayre's T-piece type anaesthetic circuit.

Figure 1 displays a Jackson Rees T-piece with a small crimp in the acute angle of the fresh gas arm.

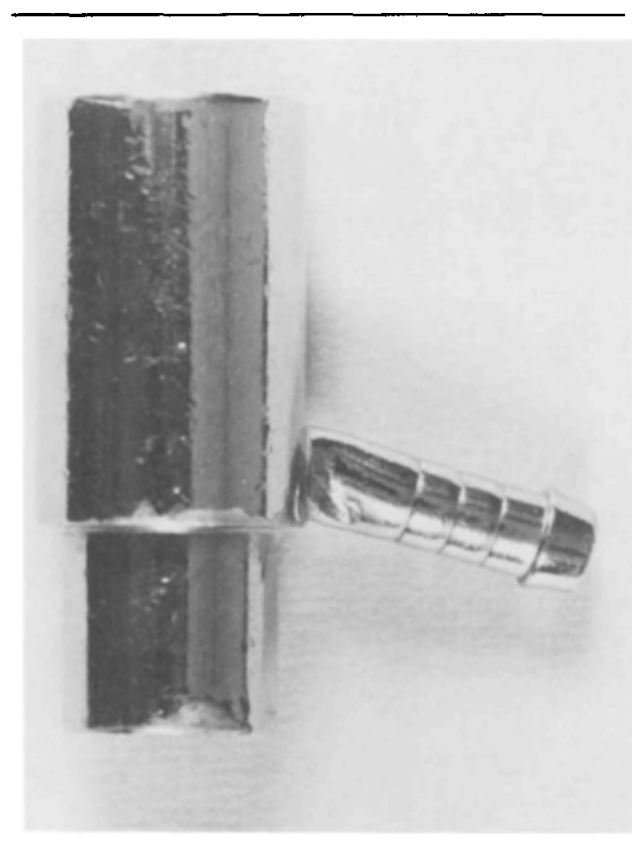

FGURE I T-piece with a small crimp in the acute angle of the fresh gas arm.

This small distortion in the T-piece may have created a site for collection of debris seen in Figure 2. This material occluded the fresh gas arm, leading to complete obstruction of fresh gas flow.

This $\mathrm{T}$-piece had been cleaned in an ultrasonic washer, it was then steam autoclaved and packaged for reuse. The material seen in Figure 2 is thought to have accummulated during this cleaning process. Microscopic examination of this debris showed it to be a disorganized fibrous substance; thus, it was not a collection of detergent but most likely some 